\title{
Campo de Dirac para fermiones sin masa
}

\author{
Jhonny R. Huamaní Chaviguri y Fulgencio Villegas Silva* \\ Facultad de Ciencias Físicas, Universidad Nacional Mayor de San Marcos, \\ Ciudad Universitaria, Av. Venezuela cdra 34, Ap. Postal 14-0149, \\ Lima 14, Perú
}

Recibido 17 julio 2010 - Aceptado 22 setiembre 2010

\begin{abstract}
Se estudia el campo de Dirac para fermiones sin masa. Considerando transformaciones quirales en el campo de Dirac se obtienen funciones de onda desacopladas en las cuales al considerar la masa de la partícula como nula se obtiene un campo que describe fermiones sin masa.

Palabras claves: Teoría cuántica de campos, física de partículas, modelo estándard.
\end{abstract}

\section{Dirac field for massless fermions}

The Dirac field of massless fermions is analyzed. Considering chiral transformations in Dirac field we obtain uncoupled wave functions in which making the mass particle null a field describing the massless fermions is obtained.

Keywords: Quantum field theory, elementary particle physics, standard model.

\section{Introducción}

Las partículas en el universo se dividen en dos grupos: los fermiones (electrones, neutrinos, quarks) y los bosones (fotones, gluones, $\mathbf{W}, \mathbf{Z}$ ). Estos grupos se distinguen por la contribución que realizan a la construcción y estabilidad del universo. Los fermiones son aquellas partículas que forman la materia, entre las cuales se ejercen fuerzas, y que manifiestan a través del operador de intercambio autoestados antisimétricos debido a sus espínes semienteros $(1 / 2,3 / 2,5 / 2, \ldots)$.

Los bosones son los propagadores que transportan las fuerzas entre fermiones, los cuales manifiestan a través del operador de intercambio autoestados simétricos debido sus espínes enteros (1 para el fotón y 2 para el gravitón). En general, para estudiar el campo fermiónico lo hacemos mediante la ecuación de Dirac, la cual es apropiada para el estudio de fermiones de espín 1/2 y la ecuación de Rarita-Schwinger para fermiones de espín $3 / 2$ la cual es una ecuación de campo relativista similar a la ecuación de Dirac.

En los últimos años se ha incrementado los trabajos acerca de fermiones sin masa, sin embargo, ya desde los años 60 surgen los primeros indícios del problema de la masa del neutrino con el experimento de R. Davis[1]. Los neutrinos originalmente se introducen en el modelo estándar, como un fermión de espín $1 / 2$, sin masa y por

\footnotetext{
*fvillegass@unmsm.edu.pe
}

lo tanto con quiralidad izquierda.

Desde el año 2004[2], el interés sobre los fermiones sin masa se ha renovado por la observación experimental de que los portadores de carga en el grafeno semejan en el punto de Dirac, donde las bandas de valencia y de conducción se superponen, a los fermiones sin masa que se mueven casi relativísticamente, con una velocidad de Fermi, $v_{F} \approx \mathrm{c} / 300$ [3]. Debido a estas extraordinarias propiedades del grafeno se ha sugerido la posibilidad de observar por primera vez en un sistema de estado sólido la paradoja de Klein, en la que las partículas relativísticas no sufren reflexión ante una barrera de potencial alta y gruesa[4].

Desde una perspectiva formativa y didáctica, en el presente trabajo analizamos el campo de Dirac en la que consideramos el caso especial en que la masa de las partículas es nula para ser tratada como una ecuación de Weyl.

\section{Campo de Dirac}

La ecuación de Dirac describe partículas fermiónicas de espín 1/2 mediante un campo fermiónico. La ecuación de Dirac para partículas de masa $m$ es[5]

$$
i \hbar \frac{\partial \psi(x)}{\partial t}=\left[c \alpha(i \hbar \nabla)+\beta m c^{2}\right] \psi(x)
$$


la cual puede ser descrita como

$$
i \hbar \gamma^{\mu} \frac{\partial \psi(x)}{\partial x^{\mu}}-m c \psi(x)=0
$$

donde

$$
\gamma^{o}=\beta, \quad \gamma^{i}=\beta \alpha_{i}, \quad i=1,2,3
$$

son las matrices de Dirac que satisfacen las relaciones de anticonmutación

$$
\left[\gamma^{\mu}, \gamma^{\nu}\right]_{+}=2 g^{\mu \nu}
$$

y las condiciones de hermiticidad $\gamma^{0 \dagger}=\gamma^{0}$ y $\gamma^{j \dagger}=-\gamma^{j}$ para $j=1,2,3$, de modo que se cumple

$$
\gamma^{\mu \dagger}=\gamma^{0} \gamma^{\mu} \gamma^{0}
$$

Si $\psi(x)$ es una función de onda espinorial con cuatro componentes $\psi_{\alpha}(x), \alpha=1,2,3,4$, el campo adjunto es definido por

$$
\bar{\psi}(x)=\psi^{\dagger}(x) \gamma^{0}
$$

y satisface la ecuación adjunta de Dirac

$$
i \hbar \frac{\partial \bar{\psi}(x)}{\partial x^{\mu}} \gamma^{\mu}-m c \bar{\psi}(x)=0 \text {. }
$$

Las ecuaciones de Dirac (2) y (6) pueden derivarse de la densidad lagrangiana

$$
L=c \bar{\psi}(x)\left[i \hbar \gamma^{\mu} \frac{\partial}{\partial x^{\mu}}\right] \psi(x)
$$

por variación de la acción respecto a los campos $\psi_{\alpha}$ y $\overline{\psi_{\alpha}}$ se obtienen los momentos conjugados

$$
\left\{\begin{array}{l}
\pi_{\alpha}(x)=\frac{\partial L}{\partial \dot{\psi}_{\alpha}}=i \hbar \psi_{\alpha}^{\dagger} \\
\bar{\pi}_{\alpha}(x)=\frac{\partial L}{\partial \dot{\bar{\psi}}_{\alpha}}
\end{array}\right.
$$

Usando las ecuaciones (7) y (8) se obtiene la densidad hamiltoniana y el momentum para el campo de Dirac, los cuales vienen expresados por

$$
H=\int d x^{3} \bar{\psi}(x)\left[-i \hbar c \gamma^{j} \frac{\partial}{\partial x^{j}}+m c^{2}\right] \psi(x)
$$

y

$$
P=i \hbar \int d x^{3} \psi^{\dagger}(x) \nabla \psi(x)
$$

respectivamente, considerando un volumen $V$ con condiciones de contorno periódicas. La ecuación de Di$\operatorname{rac}(2)$ posee cuatro soluciones independientes, éstas pueden expresarse como[6]

$$
\left\{\begin{array}{l}
u_{r}(p) \frac{e^{-i p / \hbar}}{\sqrt[2]{\mathbf{V}}} \\
\nu_{r}(p) \frac{e^{i p / \hbar}}{\sqrt[2]{\mathbf{V}}}, \quad r=1,2
\end{array}\right.
$$

donde $u_{r}(p)$ y $\nu_{r}(p)$ son espinores que satisfacen las ecuaciones

$$
\left\{\begin{array}{l}
(\not p-m c) u_{r}(p)=0 \\
\mathrm{y} \\
(\not p+m c) \nu_{r}(p)=0, \quad r=1,2
\end{array}\right.
$$

de modo que $\not p=\gamma^{\mu} p_{\mu}$.

\section{Campo de Dirac para fermiones sin masa}

Considerando en el campo de Dirac las transformaciones quirales siguientes

$$
\left\{\begin{array}{l}
\Psi(x) \rightarrow \Psi^{\prime}(x)=e^{i \alpha \gamma_{5}} \Psi(x) \\
\Psi^{\dagger}(x) \rightarrow \Psi^{\prime \dagger}(x)=e^{-i \alpha \gamma_{5}} \Psi^{\dagger}(x)
\end{array}\right.
$$

donde $\alpha$ es un parámetro real arbitrario, la densidad lagrangiana (7) reescrita como

$$
L=i \hbar c \bar{\Psi}(x) \gamma^{\mu} \partial_{\mu} \Psi(x)-m c^{2} \bar{\Psi}(x) \Psi(x)
$$

puede ser transformada, usando las ecuaciones (13) en la (14), obteniendo una densidad lagrangiana $L^{\prime}$ dada por

$$
L^{\prime}=i \hbar c \bar{\Psi}(x) \gamma^{\mu} \partial_{\mu} \Psi(x)-m c^{2} \bar{\Psi}(x) e^{2 i \alpha \gamma_{5}} \Psi(x) .
$$

Comparando las dos últimas ecuaciones, (14) y (15), se deduce que la densidad lagrangiana es invariante si la masa es nula, de modo que

$$
L^{\prime}=L=i \hbar c \bar{\Psi}(x) \gamma^{\mu} \partial_{\mu} \Psi(x)
$$

y aplicando el teorema de Euler-Lagrange a la ecuación (16) obtenemos las formas directa y adjunta

$$
\left\{\begin{array}{c}
i \hbar c \gamma^{\mu} \partial_{\mu} \Psi(x)=0 \\
i \hbar c \partial_{\mu} \bar{\Psi}(x) \gamma^{\mu}=0
\end{array}\right.
$$

respectivamente, que al aplicarles las relaciones $\left[\gamma^{5}\right]^{2}=$ 1 y $\gamma^{5}=\gamma_{5}$, obtenemos

$$
\partial_{\mu}\left[\bar{\Psi}(x) \gamma^{\mu} \gamma_{5} \Psi(x)\right]=0 .
$$

Esta última ecuación es conocida como la densidad de carga-corriente cuadrimensional $S^{\mu}$ general, pero como se obtuvo a partir de una transformacion quiral (1) ésta tiene un carácter pseudovectorial de la densidad de carga-corriente conocida como $J_{A}^{\alpha}$ con las mismas características que $S^{\mu}[7]$. Por tanto, obtenemos una densidad $J_{A}^{\alpha}$ considerando la masa nula de las partículas,

$$
J_{A}^{\alpha}=\bar{\Psi}(x) \gamma^{\alpha} \gamma_{5} \Psi(x)
$$


Sin perder generalidad, la función de onda $\Psi$ es dividida en dos componentes para un estudio más detallado, comúnmente en funciones de ondas positivas y negativas. En el caso particular en el que interviene la transformación de fase quiral, las componentes son denominadas funciones de onda de derecha e izquierda, $\Psi_{R}$ y $\Psi_{L}$, respectivamente.

$$
\Psi=\Psi_{R}+\Psi_{L}
$$

donde $\Psi_{R}$ y $\Psi_{L}$ dependen del parámetro $\alpha$ y de la matriz $\gamma^{5}$.

De la ecuación (14) y (20) obtenemos la densidad lagrangiana total para ambas componentes

$$
\begin{aligned}
L= & {\left[i \hbar c \bar{\Psi}_{R} \gamma^{\mu} \partial_{\mu} \Psi_{R}-m c^{2} \bar{\Psi}_{R} \Psi_{R}\right] } \\
& +\left[i \hbar c \bar{\Psi}_{R} \gamma^{\mu} \partial_{\mu} \Psi_{L}-m c^{2} \bar{\Psi}_{R} \Psi_{L}\right] \\
& +\left[i \hbar c \bar{\Psi}_{L} \gamma^{\mu} \partial_{\mu} \Psi_{R}-m c^{2} \bar{\Psi}_{L} \Psi_{R}\right] \\
& +\left[i \hbar c \bar{\Psi}_{L} \gamma^{\mu} \partial_{\mu} \Psi_{L}-m c^{2} \bar{\Psi}_{L} \Psi_{L}\right] .
\end{aligned}
$$

Usando las ecuaciones de Euler-Lagrange para el lagrangiano dado por la ecuación (21) obtenemos las ecuaciones de Dirac correspondientes

$$
\left\{\begin{array}{l}
i \hbar c\left[\left(\partial_{\mu} \bar{\Psi}_{R}\right) \gamma^{\mu}+\left(\partial_{\mu} \bar{\Psi}_{L}\right) \gamma^{\mu}\right]+m c^{2}\left(\bar{\Psi}_{R}+\bar{\Psi}_{L}\right)=0 \\
i \hbar c\left[\gamma^{\mu}\left(\partial_{\mu} \Psi_{R}\right)+\gamma^{\mu}\left(\partial_{\mu} \Psi_{L}\right)\right]-m c^{2}\left(\Psi_{R}+\Psi_{L}\right)=0
\end{array}\right.
$$

ordenándolos adecuadamente obtenemos

$$
\left[i \hbar \gamma^{\mu} \partial_{\mu}-m c\right] \Psi_{R}+\left[i \hbar \gamma^{\mu} \partial_{\mu}-m c\right] \Psi_{L}=0 .
$$

Reemplazando (20) en (23), se obtiene

$$
i \hbar \gamma^{\mu} \partial_{\mu} \Psi-m c \Psi=0 .
$$

Esta es conocida como la ecuación de Dirac directa, que es idéntica a la que se obtendría si se aplicara la ecuación de Euler-Lagrange directamente sobre la densidad lagrangiana general.

Usando la ecuación (24) y sumando en ambos términos $\gamma_{5}$ y realizando un artificio matemático obtenemos

$$
\begin{aligned}
& {\left[i \hbar \gamma^{\mu} \partial_{\mu}-m c\right]\left(\frac{1+\gamma_{5}}{2}\right) \Psi+} \\
& {\left[i \hbar \gamma^{\mu} \partial_{\mu}-m c\right]\left(\frac{1-\gamma_{5}}{2}\right) \Psi=0 .}
\end{aligned}
$$

Comparando las ecuaciones (23) con (25) se deduce las siguientes relaciones:

$$
\left\{\begin{array}{l}
\Psi_{R}=\left(\frac{1+\gamma_{5}}{2}\right) \Psi \\
\Psi_{L}=\left(\frac{1-\gamma_{5}}{2}\right) \Psi
\end{array}\right.
$$

donde los factores que acompañan a la función de onda son denominados operadores de proyección $\hat{P}_{ \pm}[8]$ definidos por

$$
\hat{P}_{ \pm}=\left(\frac{1 \pm \gamma_{5}}{2}\right) \text {. }
$$

Cabe mencionar que la ecuación (26) se obtuvo de la densidad lagrangiana general (14), es decir, es independiente respecto a si la partícula posee masa o no. De la ecuación (23), observamos que las funciones de onda $\Psi_{R}$ y $\Psi_{L}$ están acopladas con la masa de la partícula; es decir, una depende de la otra, por tal motivo si se desea que ambas funciones sean independientes debemos considerar que la masa de la partícula tienda a hacerse nula.

Representando la función de onda como una matriz columna tenemos

$$
\Psi=\left(\begin{array}{c}
\Psi_{L} \\
\Psi_{R}
\end{array}\right)
$$

ahora transformando la ecuación de Dirac en su forma matricial y reemplazando la ecuación (28) obtenemos las funciones de onda acopladas

$$
\left\{\begin{array}{l}
i \hbar \partial_{o} \Psi_{L}+i \hbar \bar{\sigma} \cdot \nabla \Psi_{R}=m c \Psi_{L} \\
-i \hbar \partial_{o} \Psi_{R}-i \hbar \bar{\sigma} \cdot \nabla \Psi_{L}=m c \Psi_{R} .
\end{array}\right.
$$

Tomando el límite $m \rightarrow 0$ obtenemos las funciones de onda desacopladas, conocidas también como las ecuaciones de Weyl[9, 10]

$$
\left\{\begin{array}{l}
\partial_{o} \Psi_{L}=\bar{\sigma} \cdot\left(\nabla \Psi_{R}\right) \\
\partial_{o} \Psi_{R}=-\bar{\sigma} \cdot\left(\nabla \Psi_{L}\right),
\end{array}\right.
$$

donde $\gamma^{o}$ y $\bar{\gamma}$ son estándares y $\bar{\sigma}$ es la matriz de Dirac[11].

Estas ecuaciones pudieron haberse obtenido también al considerar el límite de masa nula directamente en la densidad lagrangiana (21), de ese modo, usando las ecuaciones (17) y (26) se obtiene que

$$
\left\{\begin{array}{l}
\bar{\Psi}_{R} \Psi_{L}=\bar{\Psi}_{L} \Psi_{R}=0 \\
\bar{\Psi}_{L} \gamma^{\mu} \partial_{\mu} \Psi_{R}=\bar{\Psi}_{R} \gamma^{\mu} \partial_{\mu} \Psi_{L}=0,
\end{array}\right.
$$

considerando $m=0$ en la ecuación (21) y usando la ecuación (31) obtenemos

$$
L=i \hbar c \bar{\Psi}_{R} \gamma^{\mu} \partial_{\mu} \Psi_{R}+i \hbar c \bar{\Psi}_{L} \gamma^{\mu} \partial_{\mu} \Psi_{L} .
$$

La ecuación de Dirac para esta densidad lagrangiana es

$$
i \hbar c \gamma^{\mu} \partial_{\mu} \Psi_{R}+i \hbar c \gamma^{\mu} \partial_{\mu} \Psi_{L}=0
$$

luego, usando (33) y (31) en (32) obtenemos

$$
L=i \hbar c \bar{\Psi}_{L} \gamma^{\mu} \partial_{\mu} \Psi_{L}
$$

de la cual se obtienen las funciones de onda mano izquierda y de forma análoga mano derecha. 


\section{Conclusiones}

Para describir de manera adecuada el comportamiento de una partícula que actúa bajo una transformación quiral, ésta debe cumplir necesariamente con la condición de que la masa sea nula. Las ecuaciones de movimiento tanto de mano derecha como de mano izquierda de las funciones de onda se desligan una de la otra a partir de la consideración de masa nula, y de esta manera, ambas son independientes para describir la dinámica de cada una en particular. Las densidades lagrangianas de la ecuación (34) se ha obtenido únicamente al considerar que la partícula tenga masa nula y que se podría aplicar al caso de neutrinos.

\section{Referencias}

[1] R.J. Davis, D.S. Hamer y K.C. Hoffman, Phys. Rev. Lett. 20, 1205 (1968).

[2] K.S. Novoselov, A.K. Geim, S.V. Morozov, D. Jiang, Y. Zhang, S.V. Dubonos, I.V. Grigorieva y A.A. Firzov, Science 306, 666 (2004).

[3] A.K. Geim y K.S. Novoselov, Nature Materials 6 183 (2007).

[4] M.I. Katnelson, K.S. Novoselov y A.K. Geim, Nature Physics 2, 620 (2006).

[5] P.A.M. Dirac, Proc. Roy. Soc. A 117, 610 (1928).

[6] F. Mandl y G. Shaw, Quantum Field Theory, John Wiley \& Sons, Ltd., Londres (1993).

[7] F. Gross, Relativistic Quantum Mechanics and Field Theory, John Wiley \& Sons, Inc., Weinhein (1993).

[8] Kh. M. Beshtoev, Is neutrino produced in standard weak interactions a Dirac or Majorana particle?, arXiv:0912.0210.

[9] S.S. Schweber, An Introduction to Relativistic Quantum Field Theory, p. 108, Harper \& Row, New York, (1961).

[10] Burra G. Sidharth, The Mass of the Neutrinos, arXiv:0904.3639.

[11] W. Greiner, Relativistic Quantum Mechanics: Wave equations, tercera edición, Springer-Verlag, Berlín (2000). 